\title{
Exosomes influence the engraftment of tumor cell lines in athymic mice BALB/c nude
}

\author{
Roman A. Kovalev', Vladimir S. Burdakov' ${ }^{1}$, Elena Yu. Varfolomeeva ${ }^{1}$, Elena V. Semenova \\ and Michael V. Filatov ${ }^{1,2 *}$ \\ ${ }^{1}$ Division of Molecular and Radiation Biophysics, National Research Center "Kurchatov Institute" B.P. \\ Konstantinov Petersburg Nuclear Physics Institute; Gatchina, Russia; \\ ${ }^{2}$ Saint-Petersburg State Research Institute of Phthisiopulmonology of the Ministry of Healthcare of the \\ Russian Federation; St. Petersburg, Russia
}

\begin{abstract}
The strain of mice with immunodeficit provides a popular experimental model in oncological, immunological, and transplantation studies. Athymic nude mice (Nudes) are usually used to explore the engraftment, growth, invasive infiltration, and potency to develop metastases for different types of cancer, as well as to test in preclinical assessment new anti-tumor medical products. Nude-mutation in Foxn 1 gene results in the congenital thymus dysgenesis, and, as a consequence, in the lack of T-lymphocytes. In its turn, it leads to the suppression of the immune functions which involve T-cells. It is generally accepted that Nudes' immune deficiency provides an opportunity to engraft in them immunologically incompatible human tumors that are xenogeneic for mice.In our study we have shown that there are some types of tumors that cannot be engrafted in BALB/c Nude mice. In particular, we have discovered that tumor cells deficient in p53 gene are not able to grow in Nude mice.Furthermore, we have used in vitro model system to demonstrate that the exosomes released by the fibroblasts of BALB/c Nude mice suppress the growth of the very same tumor cell lines that cannot be engrafted in these mice, but the exosomes do not affect the growth of the tumor cells that can be engrafted successfully. One of the possible mechanisms of tumor growth suppression in BALB/c Nude mice is the transfer of the exosomes containing wild type p53 protein from the surrounding tissues to tumor cells with gene p53 damage.
\end{abstract}

KEY WORDS: BALB/C NUDE MICE, CELL LINES, EXOSOMES, PROTEIN P53

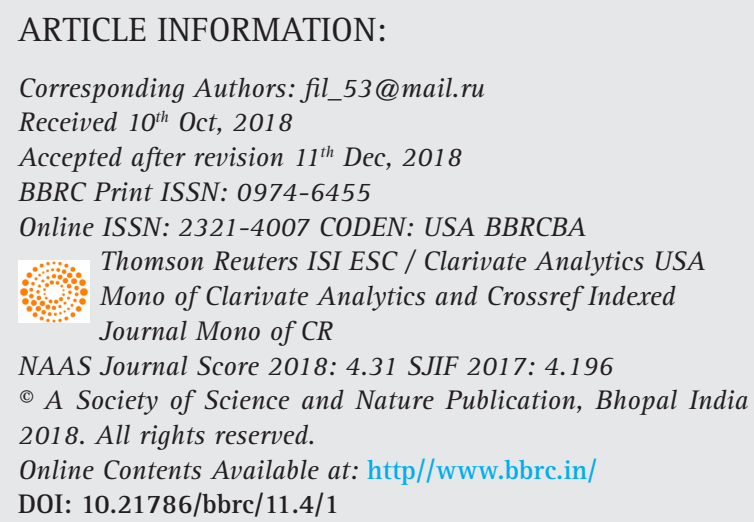




\section{INTRODUCTION}

Experimental models of human diseases play an important role both in understanding what biological and genetic factors influence phenotypic characteristics of the disease and in developing the strategy for its treatment.When using in vitro cell models for human tumor research one should take into consideration that, though these studies are necessary, they are reductionist, as often the parameters of the cell culture cannot be equated with the tumor growth inside the host's body where the tumor has specific micro-environment. Modeling human cancer with the help of genetically flawed mice is an alternative way to study carcinogenesis in vivo. It provides an opportunity to detect cell and molecular changes taking place at the initiation of cancer and during its further progression (Stakleff, 2003, Shultz, 2014; Boone, 2015).

One of the mechanisms preventing formation and growth of the malignant tumors in human body is immune system, and in particular, cell immunity based on the activity of T-lymphocytes attacking tumor cells. To research anti-tumor activity of the immune system the mice with deficient T-cell immunity are widely used. Athymic mice line BALB/c Nude is generally accepted as a model for this kind of experiments. The main advantage of this experimental model is natural immunodepression resulting from the functional deletion in Foxn 1 gene. As the result of the deletion, thymus is either deteriorated or absent, and, as a consequence, the number of T-cells is negligible, and, therefore, cell immunity is inhibited (Pignata,1996; Frank, 1999; Zuklys, 2016). In cancer research a large number of various tumor cell lines are used for inoculation hence, it is comparatively easy to engraft a tumor being studied in the mice just inoculating the host-animal with the tumor cells. It is believed that BALB/c Nude mice can host even immunologically incompatible, xenogneic for mice human tumors, (Liebman, 2007; Zeineldin, 2014; Conrad, 2015).

However, in our study we discovered that not all tumor grafts could grow in BALB/c Nude immune deficient mice. We were not able to reach a positive result of inoculation with primary glioma lines developed in our laboratory. The fact led us to the idea of an alternative mechanism of tumor growth inhibition - the mechanism which did not involve the immune system. If the mechanism exists, then even though Nude mice lack T-lymphocyte mediated cell immunity, the mechanism will account for inhibition of the growth of inoculated human tumor cells.Taking into consideration the growing amount of the data that point to participation of exosomes in formation, development and inhibition of cancer (Webber, 2015; Yu, 2015), we suggested that the discovered phenomenon of tumor growth inhibition in BALB/c Nude mice was possibly a result of the influence of the exosomes formed in the tissues surrounding the tumor.

Exosomes are extracellular membrane nano vesicles that are released from multivesicle bodies into extracellular space through exocytosis (Huang, 2013). The ability of the exosomes to be efficient transporters of exogenous proteins and RNA into cells-recipients has been reported (Shtam, 2013; Haney, 2015; Srivastava, 2016). Earlier in our experiments in vitro we demonstrated that the exosomes released from the cells, which contained wild type of the p53 protein, were able to inhibit the growth of the cancer cells lacking the same protein. Furthermore, the exosomes both released by the cell lines and isolated from human blood plasma carried one of the main tumor suppressors - protein p53 (Burdakov, 2017; Jorgensen, 2015, Burdakov, 2017).

In this study we now present some arguments in favor of our assumption that one of the factors inhibiting inoculated tumor growth in BALB/c Nude athymic mice may be negative effect of exosomes on tumor cells. The effect may be based on the reaction of the inoculated tumor cells deficient in $p 53$ gene to the wild type p53 protein, which exists in exosomes released by the surrounding tissues.

\section{MATERIALS AND METHODS}

\section{CELL LINES AND CULTIVATION CONDITIONS}

In our study we used both transplantable and primary human cell lines: HT-1080 (fibrosarcoma), Gl-V, Gl-R, Gl-Sh (primary cultures of glioma cells, developed in our laboratory), transplantable culture of rat cells C6 (rat brain glioma), and fibroblasts of BALB/c Nude mice. Cells were cultivated in DMEM/F12 medium (Biolot, Russia) supplemented with 10\% fetal bovine serum (Biolot, Russia), without antibiotics, under $5 \% \mathrm{CO}_{2}$, at $37^{\circ} \mathrm{C}$. When tumor cells of each of the lines were cultivated with isolated exosomes, the exosomes had final concentration in the culture of $10^{13}-10^{14}$ exosomes $/ \mathrm{ml}$.

\section{Isolation of exosomes in the systems in vitro}

Conditional medium was collected from growing cell culture. Then, it was consequently centrifuged at 2,000 $\mathrm{g}$ and at 20,000 g to eliminate dead cells and their fragments. The procedure was repeated until the amount of the purified conditional medium reached $500 \mathrm{ml}$. This amount of collected purified conditional medium was used to isolate exosomes by ultracentrifuging. Beckman coulter ultracentrifuge was used (45Ti rotor) at 100,000 g for 2 hours. After that, the residue was suspended in $100 \mathrm{ml}$ of PBS and underwent the second ultracentrifugation at the same conditions. The exosomes obtained as the result of the second sedimentation were used in the experiments. 


\section{Evaluation of the cell survival rate}

The cells were suspended in Versene/Trypsin solution (Biolot, Russia), and the survival rate was evaluated through direct calculation of the cells with the help of vision-based automated cell counter Scepter (Millipore, USA). To visualize cell survival, the cells of Gl-V, Gl-R, Gl-Sh and C6 lines were seeded into 12-well plates in the concentration equal for all the cell lines $(10,000$ cells per a well). After that we added equal amounts of the exosomes harvested from the conditional medium of the fibroblasts of BALB/c Nude mice to all the cell lines. As the control cells formed a monolayer, all the cells in the well plates were stained with Crystal Violet (Fluka AG, Germany). The data were analyzed by Student's t test. Data are shown as the mean \pm SEM (standard error of the mean).

\section{Animal care and engraftment rate evaluation}

Adult BALB/c Nude mice, 7-8 weeks of age and 18-20 grams of bodyweight, were purchased from the laboratory animal breeding center "Pushchino" (Pushchino, Russia). All procedures for mouse care and use were conducted in accordance with the National Standard of the Russian Federation GOST R 53434-2009: Principles of good laboratory practice (introduced 01.03 .2010 by Federal Agency for Technical Regulation and Metrology, published by Standardinform, Moscow, 2010). The protocol was approved by scientific committee of the Division of Molecular and Radiation Biophysics of National Research Center "Kurchatov Institute" B.P.Konstantinov St Petersburg Nuclear Physics Institute (Gatchina, Russia).

Animals were housed in groups of three per cage (males and females were housed separately) with ad libitum access to food and water in standard polycarbonate cages (width: $300 \mathrm{~mm}$, depth: $400 \mathrm{~mm}$, height: $200 \mathrm{~mm}$ ) and maintained under standard conditions of temperature $\left(22 \pm 2^{\circ} \mathrm{C}\right)$, humidity $(55 \pm 10 \%)$, artificial 12 -hour light-dark cycle (lights on at 8:00 A.M., lights off at 8:00 P.M.). The mice were fed on the nutrient extruded and granulated food designed for experimental rodents (LLC "Laboratorkorm», Russia).

Tumor inoculations were performed through intramuscularly injections of 150,000 to $1,000,000$ tumor cells. To estimate the engraftment rate for each tumor cell line, not less than $20 \mathrm{BALB} / \mathrm{c}$ Nude immune deficient athymic mice were used.

\section{RESULTS AND DISCUSSION}

\section{Evaluation of engraftment in BALB/c Nude immune deficient athymic mice for tumor cell lines}

To evaluate the engraftment rate we took 5 different tumor cell lines, which were cultivated in our laboratory.
Two of them were standard transplantable cell lines: HT-1080, C6. The rest three lines (GL-V, GL-R, GL-Sh) were primary lines of human gliomas, developed in our laboratory. In the experiments with each of the cell lines not less than $20 \mathrm{BALB} / \mathrm{c}$ Nude immune deficient athymic mice were used. In the first experiment engraftment was performed through intramuscularly injections of 150,000 tumor cells per animal. As the result there were two polar outcomes: transplantable tumor lines HT-1080 and C6 engrafted in 100\% of the animals; tumor lines GL-V, GL-R did not engraft at all (fig. 1). Engraftment rate of the latter lines (GL-V, GL-R) did not change with the increase in the amount of inoculated cells up to $1,000,000$ cells per animal.

\section{Analysis of survival rate of the tumor cell lines after treatment with the exosomes from the fibroblasts of BALB/c Nude mice}

We assumed that negative results of engraftment of some of the tumors in BALB/c Nude athymic mice stemmed from the ability of exosomes to inhibit tumor cell growth. To confirm the assumption, we decided to compare the engraftment rate obtained in the experiments with the survival rate of the same tumor cell lines after they had been treated with the exosomes from the fibroblasts of BALB/c Nude mice. To carry out the comparison, we used standard ultracentrifugation methods described previously, (Lässer et al., 2012 and Shtam et al 2013) to isolate exosomes from the condensed cultural medium after growing fibroblasts of BALB/c Nude mice in it. Cells from the analyzed lines were seeded onto 24-well plates in the equal concentration. Then, equal amounts of the isolated exosomes were added to all of the tested tumor cell lines. In 10 days after the exosomes had been added, the number of living cells was calculated for each of the studied tumor cell lines.

The results of the calculation are presented in fig. 2 . Addition of the exosomes isolated from the fibroblasts of BALB/c Nude mice did not affect the survivability of the tumor cells HT-1080 and GL-Sh. However, exosome addition to the cells of the tumor lines GL-R и GL-V resulted in significant decrease in their survivability (fig. 2). Survivability of the glial cells of GL-R cell line decreased by almost three times, and survivability of the cells of the cell line GL-V decreased by more than 10 times. The obtained results point to the ability of the BALB/c Nude cell exosomes to inhibit the growth of the target cells GL-R and GL-V in in vitro experiments. It correlates with the negative results of in vivo inoculation of the cells of these tumor lines to BALB/c Nude immune deficient mice.

Exosomes, participating in inter-cellular communication by transporting RNA and specific proteins between cells, are able to cause both functional and epigenetic 


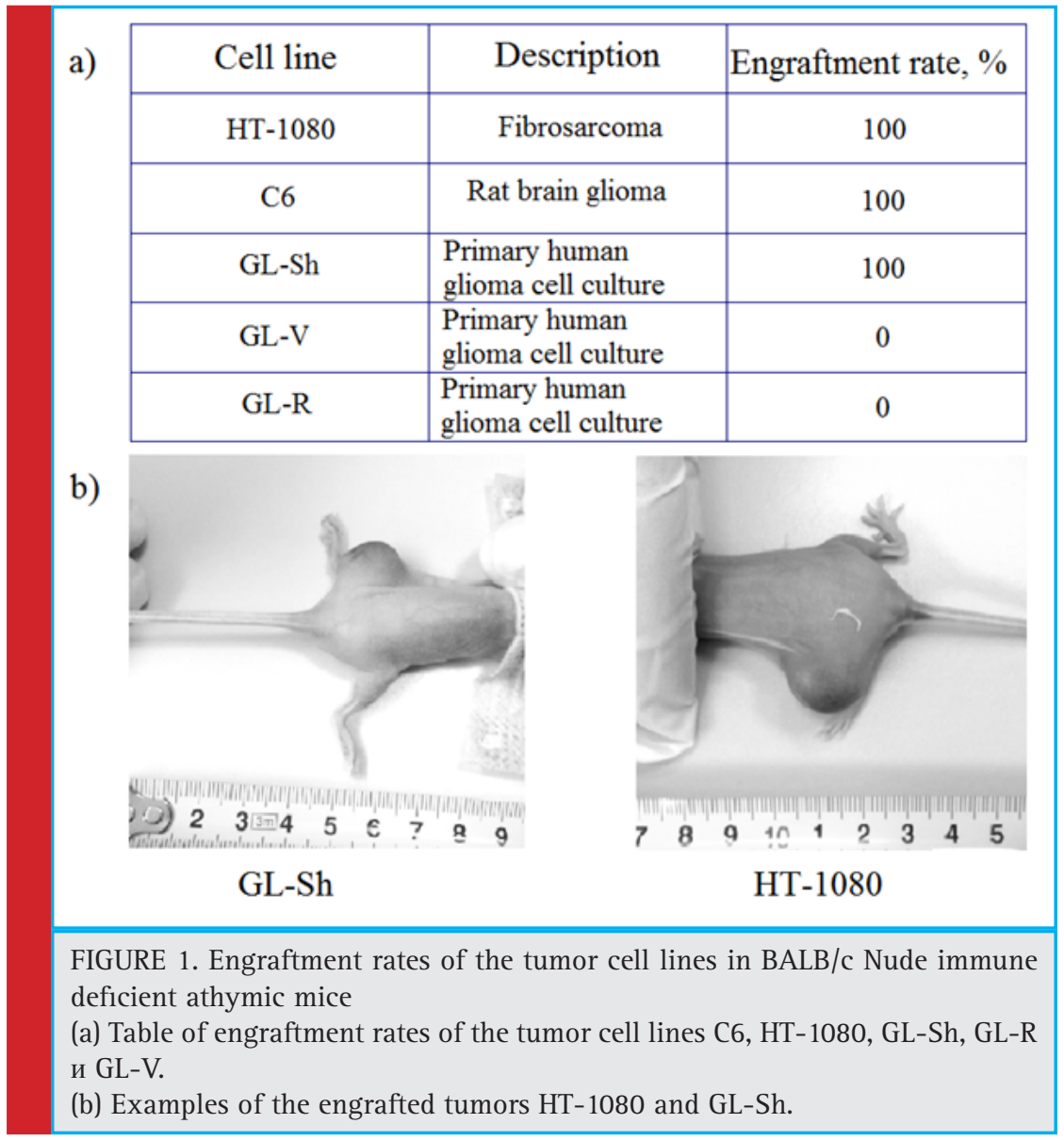

changes in the recipient cells (Grange, 2011; Kobayashi, 2014). Both discovery of p53 oncosupressor in the exosomes of the human blood plasma and their ability to affect proliferation of p53-negative cells confirm their important role in control over oncogenesis (Burdakov, 2017; Jorgensen, 2015). Protein p53 is the main par- ticipant of many signaling pathways through which cell reactions to stress are regulated. The protein is able to stop cell-division cycle and/or to cause apoptosis, thus preventing uncontrollable cell division - reproduction of the cells with damaged genome. In human malignant tumors p53 gene is the one that most often is found mutated

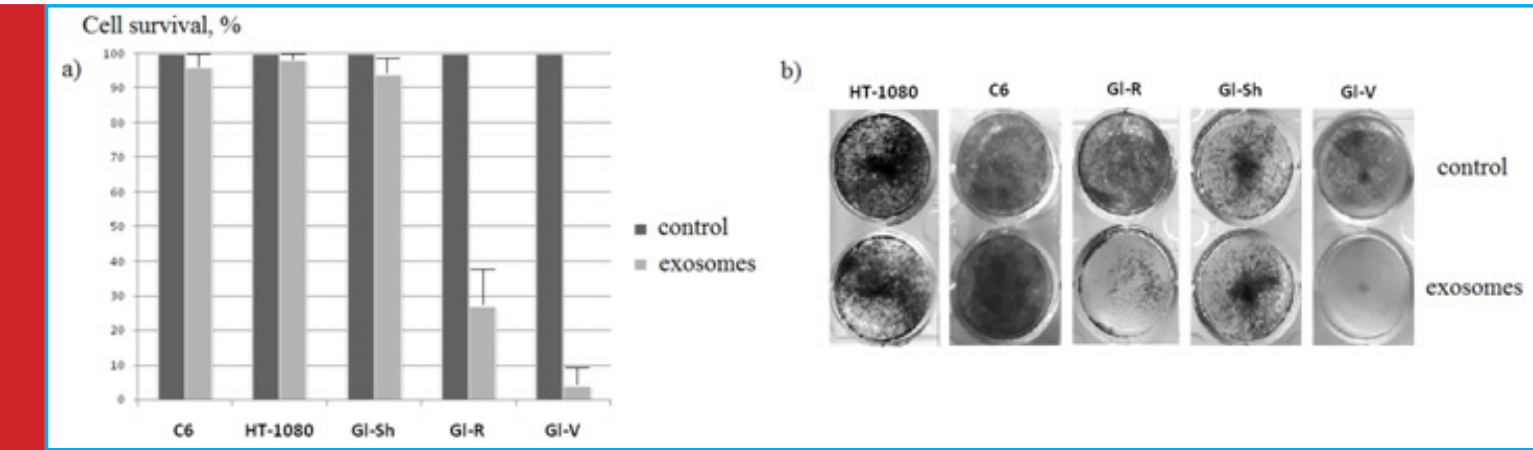

FIGURE 2. Survival rates of the tumor cell lines C6, HT-1080, GL-Sh, GL-R and GL-V after treatment with the exosomes from the fibroblasts of BALB/c Nude mice

(a) Histograms of survival rates of the tumor cell lines after treatment with the exosomes from the fibroblasts of BALB/c Nude mice.

(b) Survival of the cell lines C6, HT-1080, GL-Sh, GL-R and GL-V after coculturing with the exosomes from the fibroblasts of BALB/c Nude mice demonstrated by means of Crystal Violet staining. 
(Levine, 2009; Vogelstein, 2013; Kandoth, 2013). There are a number of mouse model studies demonstrating that restoration of the function of wild type p53 may result in tumor regression (Ventura, 2007; Xue, 2007).

We assume that negative effect of the exosomes containing wild type p53 protein on inoculated tumor cells may be one of the factors which prevent the engraftment of the tumor in BALB/c Nude athymic mice.

It is a common knowledge that any experimental model based on the usage of the athymus Nude mice has its restrictions: immunodeficiency is severe, but not absolute (there still exists humoral adaptive immune system and undamaged congenital immunity). Hence, despite almost complete lack of functioning T-lymphocytes, both congenital immune response and high activity of NK-cells are able to restrict the speed of engraftment and to decrease metastatic potency of majority of tumors (Shultz, 2005; Shultz, 2014; Szadvari, 2016).

However, the data on the engraftment rate of different tumor cell lines we present in this study point to a clear division of the tumors into two groups: the tumors engrafting in BALB/c Nude mice and the tumors which cannot be engrafted in BALB/c Nude mice. Basing on the vast statistics we discovered no variations in the engraftment rate for each of the tumors used in the study (fig.1a). These facts cannot be explained only by existence of the rudimentary immunity in immune deficient mice. We assume that one of the possible mechanisms of the engraftment inhibition involves systemic influence of mouse's own exosomes on the inoculated tumor cells. It is necessary to remark that the cells with the wild type of p53 protein (HT-1080) had 100\% engraftment rate, while GL-V cells lacking endogenous p53 protein (Kovalev, 2015) could not be engrafted at all.

Then we examined the sensitivity of all the tumor cell lines used for inoculation to the influence of the exosomes isolated from the fibroblasts of BALB/c Nude mice. The results we received in the experiments in vitro showed that cells of the GL-R cell line and especially p53-negative GL-V line were sensitive to such influence: the cells from abovementioned tumor cell lines died as the result of addition of the exosomes from the fibroblasts of BALB/c Nude mice to the tumor cell culture.The results obtained support the hypothesis that exosomes circulating in the body are able to perform a defense function controlling oncogenesis through onco suppressor p53.

Conflict of interest disclosures: We have no conflict of interest in any part of this article.

\section{REFERENCES}

Boone JD, Dobbin ZC, Straughn JM jr., Buchsbaum DJ: (2015) Ovarian and cervical cancer patient derived xenografts: The past, present, and future. Gynecol Oncol 138: 486-491.
Burdakov VS, Kovalev RA, Pantina RA, Varfolomeeva EYu, Makarov EM., Filatov MV: (2017) Exosomes transfer p53 between cells and can suppress growth and proliferation of p53-dR. Tsitologiia 59 (9): 588-594.

Conrad SJ, El-Aswad M, Kurban E, Jeng D, Tripp BC, Nutting C, Eversole R, Mackenzie C, Essani K: (2015) Oncolytic tanapoxvirus expressing FliC causes regression of human colorectal cancer xenografts in nude mice. J Exp Clin Cancer Res 34 (1): 19.

Frank J, Pignata C, Panteleyev AA, Prowse DM, Baden H, Weiner L, Gaetaniello L, Ahmad W, Pozzi N, Cserhalmi-Friedman PB, Aita VM, Uyttendaele H, Gordon D, Ott J, Brissette JL, Christiano AM: (1999) Exposing the human nude phenotype. Nature 398 (6727): 473-474.

Grange C, Tapparo M, Collino F, Vitillo L, Damasco C, Deregibus MC, Tetta C, Bussolati B, Camussi G: (2011) Microvesicles released from human renal cancer stem cells stimulate angiogenesis and formation of lung premetastatic niche. Cancer Res 71: 5346-5356.

Haney M J, Klyachko NL, Zhao Y, Gupta R, Plotnikova EG, He Z, Patel T, Piroyan A, Sokolsky M, Kabanov AV, Batrakova EV: (2015) Exosomes as drug delivery vehicles for Parkinson's disease therapy. J Control Release 207: 18-30.

Huang SH, Li Y, Zhang J, Rong J, Ye S: (2013) Epidermal growth factor receptor-containing exosomes induce tumorspecific regulatory T cells. Cancer Invest 31: 330-335.

Jørgensen MM, Bæk R, Varming K: (2015) Potentials and capabilities of the extracellular vesicle (EV) array. J Extracell Vesicles 4: 26048.

Kandoth C, McLellan MD, Vandin F, Ye K, Niu B, Lu C, Xie M, Zhang Q, McMichael JF, Wyczalkowski MA, Leiserson MDM, Miller CA, Welch JS, Walter MJ, Wendl MC, Ley TJ, Wilson RK, Raphael BJ, Ding L: (2013) Mutational landscape and significance across 12 major cancer types. Nature 502: 333339.

Kobayashi M, Salomon C, Tapia J, Illanes SE, Mitchell MD, Rice GE: (2014) Ovarian cancer cell invasiveness is associated with discordant exosomal sequestration of Let-7 miRNA and miR-200. J Transl Med 12: 4.

Kovalev RA, Shtam TA, Karelov DV, Burdakov VS, Volnitskiy AV, Makarov EM, Filatov MV: (2015) Histone deacetylase inhibitors cause the TP53-dependent induction of p21/Waf1 in tumor cells carrying mutations in TP53. Tsitologiia 57 (3): 204-211.

Lässer C, Eldh M, Lötvall J: (2012) Isolation and characterization of RNA-containing exosomes. J Vis Exp 59: e3037.

Levine AJ, Oren M: (2009) The first 30 years of p53: growing ever more complex. Nat Rev Cancer 9: 749-758.

Liebman MA, Roche MI, Williams BR, Kim J, Pageau SC, Sharon J: (2007) Antibody treatment of human tumor xenografts elicits active anti-tumor immunity in nude mice. Immunol Lett 114 (1): 16-22.

Pignata C, Fiore M, Guzzetta V, Castaldo A, Sebastio G, Porta F, Guarino A: (1996) Congenital Alopecia and nail dystrophy 
associated with severe functional T-cell immunodeficiency in two sibs. Am J Med Genet 65 (2): 167-170.

Shtam TA, Kovalev RA, Varfolomeeva EYU, Makarov EM, Kil YUV, Filatov MV: (2013) Exosomes are natural carriers of exogenous siRNA to human cells in vitro. Cell Commun Signal 11: 88.

Shultz LD, Goodwin N, Ishikawa F, Hosur V, Lyons BL, Greiner DL: (2014) Human cancer growth and therapy in NOD/ SCID/IL2R $\gamma$ null (NSG) mice. Cold Spring Harb Protoc 694708.

Shultz LD, Lyons BL, Burzenski LM, Gott B, Chen X, Chaleff S, Kotb M, Gillies SD, King M, Mangada J, Greiner DL, Handgretinger R: (2005) Human lymphoid and myeloid cell development in NOD/LtSz-scid IL2R $\gamma$ null mice engrafted with mobilized human hemopoietic stem cells. J Immunol 174: 6477-6489.

Srivastava A, Babu A, Filant J, Moxley KM, Ruskin R, Dhanasekaran D, Sood AK, McMeekin S, Ramesh R: (2016) Exploitation of exosomes as nanocarriers for gene-, chemo-, and immune-therapy of cancer. J Biomed Nanotechnol 12: 11591173.

Stakleff KDS, von Gruenigen VE: (2003) Rodent models for ovarian cancer research. Int J Gynecol Cancer 13: 405-412.

Szadvari I, Krizanova 0, Babula P: (2016) Athymic nude mice as an experimental model for cancer treatment. Physiol Res 65 (Supplementum 4): 441-453.
Ventura A, Kirsch DG, McLaughlin ME, Tuveson DA, Grimm J, Lintault L, Newman J, Reczek EE, Weissleder R, Jacks T: (2007) Restoration of p53 function leads to tumour regression in vivo. Nature 445: 661-665.

Vogelstein B, Papadopoulos N, Velculescu VE, Zhou S, Diaz LA jr, Kinzler KW: (2013) Cancer genome landscapes. Science 339: 1546-1558.

Webber J, Yeung V, Clayton A: (2015) Extracellular vesicles as modulators of the cancer microenvironment. Semin Cell Dev Biol 40: 27-34.

Xue W, Zender L, Miething C, Dickins RA, Hernando E, Krizhanovsky V, Cordon-Cardo C, Lowe SW: (2007) Senescence and tumour clearance is triggered by p53 restoration in murine liver carcinomas. Nature 445: 656-660.

Yu S, Cao H, Shen B, Feng J: (2015) Tumor-derived exosomes in cancer progression and treatment failure. Oncotarget 6: 37151-37168.

Zeineldin M, Jensen D, Paranjape SR, Parelkar NK, Jokar I, Vielhauer GA, Neufeld KL: (2014) Human cancer xenografts in outbred Nude mice can be confounded by polymorphisms in a modifier of tumorigenesis. Genetics 197 (4): 1365-1376.

Zuklys S, Handel A, Zhanybekova S, Govani F, Keller M, Maio S, Mayer CE, Teh HY, Hafen K, Gallone G, Barthlott T, Ponting CP, Holländer GA: (2016) Foxn1 regulates key target genes essential for T cell development in postnatal thymic epithelial cells. Nat Immunol 17: 1206-1215. 